\title{
Postoperative Complications After Extracapsular Dissection of Benign Parotid Lesions With Particular Reference to Facial Nerve Function
}

\author{
Nils Klintworth, MD; Johannes Zenk, MD, PhD; Michael Koch, MD; Heinrich Iro, MD, PhD
}

Objectives/Hypothesis: The desirable extent of surgical intervention for benign parotid tumors remains a matter of controversy. Superficial or total parotidectomy as a standard procedure is often said to be the gold standard; however, with it the risk of intraoperative damage to the facial nerve cannot be ignored. For some time now, extracapsular dissection without exposure of the main trunk of the facial nerve has been favored as an alternative for the treatment of discrete parotid tumors. Data on the incidence of facial nerve lesions and other acute postoperative complications of extracapsular dissection have been lacking until now.

Study Design: Retrospective analysis.

Methods: We performed a retrospective analysis of the data from patients in whom extracapsular dissection of a benign parotid tumor had been performed under facial nerve monitoring and as a primary intervention in our department between 2000 and 2008 .

Results: A total of 934 patients were operated on for a newly diagnosed benign tumor of the parotid gland. Three hundred seventy-seven patients (40\%) underwent extracapsular dissection as a primary intervention. The most common postoperative complication was hypoesthesia of the cheek or the earlobe, as reported by 38 patients (10\%). Eighteen patients (5\%) developed a seroma and 13 patients $(3 \%)$ a hematoma. A salivary fistula formed in eight patients $(2 \%)$. Secondary bleeding occurred in three patients $(0.8 \%)$. In 346 patients $(92 \%)$ facial nerve function was normal (House-Brackmann grade I) in the immediate postoperative period, whereas 23 patients $(6 \%)$ showed temporary facial nerve paresis (House-Brack-

From the Department of Otorhinolarnygology, Head and Neck Surgery, University of Erlangen-Nuremberg, Erlangen, Germany.

Editor's Note: This Manuscript was accepted for publication November 6, 2009.

Send correspondence to Heinrich Iro, $\mathrm{MD}, \mathrm{PhD}$, Chairman of the Department of Otorhinolarnygology, Head and Neck Surgery, University of Erlangen-Nuremberg, Waldstrasse 1, D-91054 Erlangen, Germany. E-mail: heinrich.iro@uk-erlangen.de

DOI: 10.1002/lary.20801 mann grade II or III) and eight patients (2\%) developed permanent facial nerve paresis (seven patients House-Brackmann grade II, one patient House-Brackmann grade III).

Conclusions: Extracapsular dissection of benign parotid tumors is associated with a low rate of postoperative complications, a fact that is confirmed by the available literature. We therefore recommend that use of this technique always be considered as a means of treating benign parotid tumors as conservatively, that is, as uninvasively, as possible.

Key Words: Parotid tumors, parotidectomy, extracapsular dissection, facial nerve paresis.

Laryngoscope, 120:484-490, 2010

\section{INTRODUCTION}

About $80 \%$ of parotid tumors are benign. Of these, the most common histological subtype is pleomorphic adenoma, which accounts for $65 \%$, followed by adenolymphoma or Warthin's tumor, which accounts for about $25 \%$. Other adenomas, such as basal cell adenomas and oncocytomas, are far less common. ${ }^{1}$

In the first half of the twentieth century benign parotid tumors were often treated by (intracapsular) enucleation, that is, the tumor was exposed, the tumor capsule opened, and the tumor tissue lifted out of the capsule in its entirety. ${ }^{2,3}$ Because with this technique capsular structures were left in situ and tumor cells could be distributed over the operative field, the rate of tumor recurrence was relatively high-according to some reports up to $20 \%$ to $45 \%^{2,4,5}$ - and a need for a better surgical technique was perceived.

Over the following decades the technique of parotidectomy was refined first in the sense that the tumor was removed in toto with surrounding glandular tissue, and second that the facial nerve was fully dissected using an anterograde or retrograde approach. ${ }^{5,6}$ This resulted in a lateral or superficial parotidectomy if only the outer part of the gland, namely, the part lateral to the facial nerve, was removed or a total parotidectomy if 
the inner part of the gland was also removed. This now largely standard operative technique is still regarded by many as the treatment of choice for benign parotid tumors. The recurrence rate of $0 \%$ to $5 \%$ reported with use of this technique is regarded as acceptable ${ }^{7,8}$; however, the dissection of the facial nerve and its branches and the removal of large parts of the gland can lead to postoperative complications that cannot be ignored. For example, the rates of temporary and permanent facial nerve paresis are reported to be $15 \%$ to $25 \%$ and $5 \%$ to $8 \%$, respectively, after superficial parotidectomy and as high as $20 \%$ to $50 \%$ and $5 \%$ to $10 \%$, respectively, after total parotidectomy. ${ }^{9-11}$ There is also a risk of Frey's syndrome, the incidence of which has been reported by some authors to be over $10 \%$ after superficial parotidectomy and over $30 \%$ after total parotidectomy. ${ }^{7,12,13}$

As these rates of important postoperative complications must be regarded as very high for the treatment of a benign condition, over the past 20 years a number of techniques of partial resection of the parotid gland have been described, in which less than the entire superficial part of the gland is removed and the facial nerve is dissected only in part or not at all. ${ }^{12,14,15}$ This has resulted in markedly lower rates of facial nerve paresis and in particular also of Frey's syndrome. At the same time, the first long-term observations to be published show no increase in recurrence rate after circumscribed resections of this type. ${ }^{12,16,17}$

The present article shows the results obtained in our department for the occurrence of postoperative complications after extracapsular dissection of benign parotid tumors in the period 2000 to 2008. We report retrospectively on data from 377 patients who underwent an extracapsular dissection in this period and who could be followed up consistently. This is the largest group of patients to have been reported on to date.

\section{MATERIAL AND METHODS}

\section{Patients Included and Data Collected}

We performed a retrospective analysis of all patients with the diagnosis of a benign tumor of the parotid gland who were operated on in our department (Ear, Nose and Throat Department, Head and Neck Surgery, University Hospital Erlangen, Erlangen, Germany) in the period 2000 to 2008. An additional analysis included only those patients who underwent extracapsular dissection (see below) as a primary intervention (i.e., had not undergone any previous operation on the parotid gland concerned). Data collected included general data, such as patient age and gender, as well as a number of specific clinical and imaging investigations.

We indicated extracapsular dissection as surgical procedure in cases of a single and mobile benign lesion located superficially within the lateral lobe of the parotid gland. Other entities were treated by parotidectomies of variable extent.

\section{Preoperative Investigations}

After detailed questioning about the nature and duration of their symptoms, patients underwent a clinical examination including full ear, nose, and throat endoscopy. An ultrasound scan of the head and neck with particular attention to the parotid glands was carried out in all patients. Additional imaging investigations, such as computed tomography and magnetic resonance imaging scans, were dispensable because the lesions treated by extracapsular dissection are located in the superficial part of the parotid gland and can be thoroughly examined sonographically.

Preoperatively, the function of the facial nerve was assessed clinically using the House-Brackmann grading system $^{18}$ and by electromyography.

\section{Intraoperative Procedure}

Descriptions of the surgical technique used for partial resection of the parotid gland vary greatly and consequently are confusing. We therefore propose the adoption of a standard classification of techniques based on the extent of dissection of the facial nerve. According to this classification, procedures in which the main trunk of the facial nerve is exposed but only part of the superficial lobe of the parotid gland is removed are referred to as "partial parotidectomy." This technique is closely related to superficial parotidectomy, in which the trunk of the facial nerve and its branches are likewise dissected. By contrast, we would use the term "extracapsular dissection" to refer to cases in which the tumor is removed without exposure of the main trunk of the facial nerve. On no account should the term "enucleation" be used to refer to this type of operation, because this could cause confusion with the historical technique of enucleation referred to above.

The surgical preparation (including facial nerve monitoring, see below) for extracapsular dissection are the same as for conventional parotidectomy. Here too a curved skin incision around the earlobe is made starting at the tragus and ending in a skin fold in the neck (Fig. 1a). The skin flap and subcutaneous tissue are then dissected and the anterior border of the sternocleidomastoid muscle, the great auricular nerve, and finally the capsule of the parotid gland are exposed (Fig. 1b). Before the parotid capsule is opened the tumor is once again palpated. If the exact position of the tumor cannot be determined, an ultrasound scan can be performed intraoperatively. The parotid capsule is now incised and the dissection extended toward the tumor; however, the tumor capsule itself is never opened (Fig. 1c). The dissection is now extended through the healthy glandular tissue around the tumor so as to gradually separate the tumor from the gland, care being taken at all times to dissect away from the tumor (Fig. 1d). With this technique a rim of about 2 to $3 \mathrm{~mm}$ of healthy glandular tissue is left on the tumor, without damaging the facial nerve as far as possible. After the tumor has been removed the parotid capsule is sutured back together. A rubber or Redon drain is inserted and subcutaneous and skin sutures applied. A pressure bandage is then 


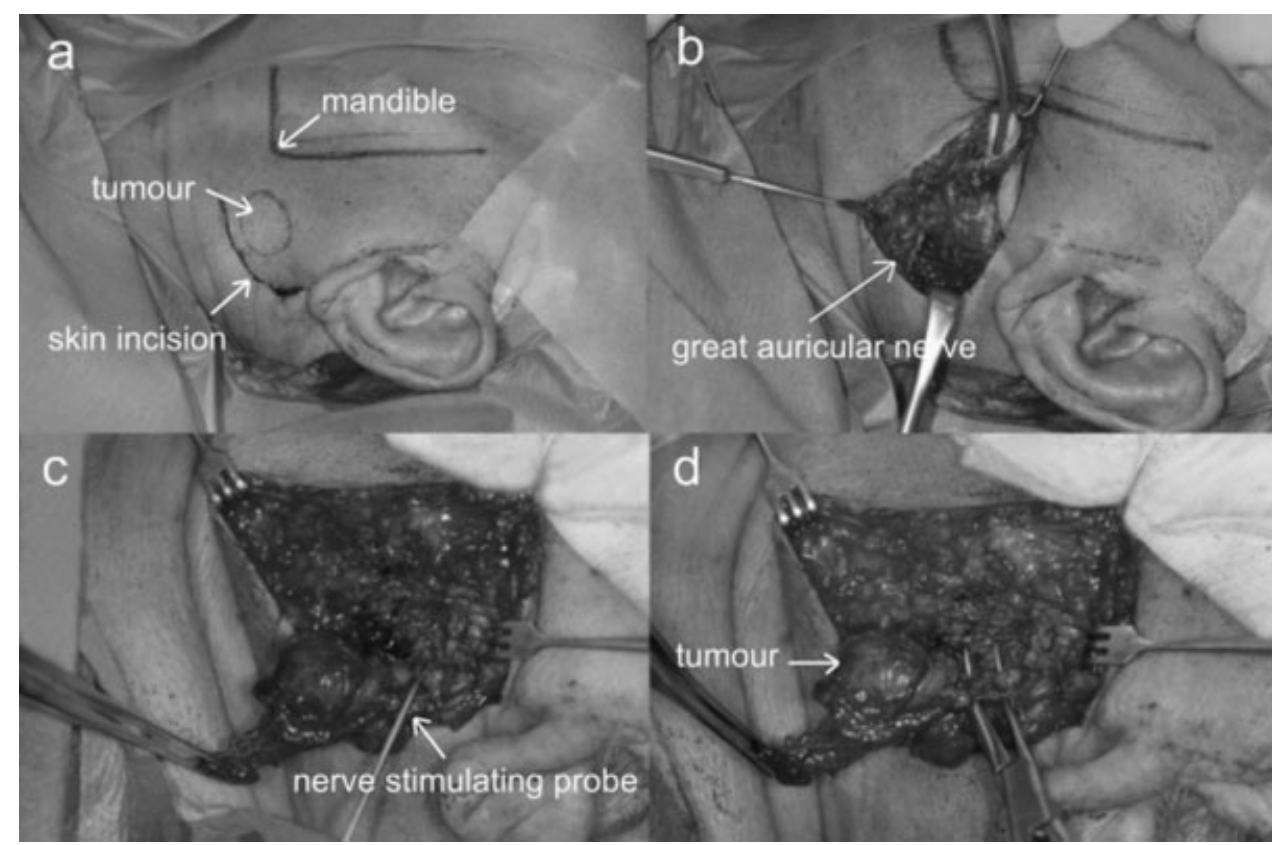

Fig. 1. (a) Marking of tumor and mandible before skin incision around the earlobe. (b) Preparation of skin flap and subcutaneous tissue with preservation of the great auricular nerve. (c) Use of facial nerve monitoring (stimulation probe) during dissection of glandular tissue. (d) Direction of preparation is always off the tumor when separating it from the gland. applied; this is changed daily and kept in place for 5 to 7 days.

To identify and protect the branches of the facial nerve, mandatory neuromonitoring is performed (Fig. 1c). This consists of an electrical stimulation probe and two electrodes for conducting the action potentials of the orbicularis oculi and orbicularis oris muscles. In the beginning, stimulation is carried out with a maximum current of $5 \mathrm{~mA}$. As soon as a branch of the facial nerve is identified by positive stimulation, the stimulating current is reduced to $2 \mathrm{~mA}$. After exposure of a branch of the facial nerve, the current is reduced further to $1 \mathrm{~mA}$ or less. Nerve branches are exposed only if they are situated close to the tumor and definite identification of them is considered desirable before further dissection of the tumor. The main trunk of the facial nerve, on the other hand, is not exposed during extracapsular dissection (see definition above).

\section{Postoperative Management}

A pressure bandage was applied immediately after the operation. This was changed daily over the first few days. Antibiotic therapy was not given routinely.

Patients were asked about the presence of pain and any sensory disturbances and their wounds were assessed daily, first clinically, and sonographically if any abnormalities were detected to identify or exclude fluid accumulation in the form of a seroma or hematoma. Any such fluid accumulations were drained locally, and local antiseptic therapy and systemic antibiotic therapy was given if necessary.

The function of the facial nerve was once again assessed clinically using the House-Brackmann scale. Patients in whom facial nerve dysfunction was detected then underwent further investigation in the form of clinical and electromyographic examination at regular intervals (after 2 weeks, 3 months, 6 months and 1 year). In particular, this made it possible to document the evolution of the lesion and thus to determine whether the facial nerve paresis was temporary or permanent.

Patients were followed up via regular visits to our outpatients' department or the attending physician so that even late complications could be detected.

\section{RESULTS}

\section{General and Epidemiological Data}

Between the years 2000 and 2008 a total of 377 patients underwent extracapsular dissection of a benign parotid tumor as a primary intervention in our department. This corresponds to $40.4 \%$ of the 934 primary operations that were performed for benign parotid tumors in the same period (Table I). The proportion of patients in whom the surgical technique of extracapsular dissection was used increased from $13 \%$ in 2000 to $57 \%$ in 2008. The 377 patients consisted of 308 women and 69 men aged between 7 and 91 years with a mean age of 54.65 years.

\section{Histology}

All the parotid tumors considered here were benign. The distribution of histological entities was as follows (Table II): 124 pleomorphic adenomas (32.9\%), 128 Whartin's tumors $(34.0 \%), 44$ other benign tumors (11.7\%), and 81 other benign lesions (21.5\%) including particularly cysts and lymph nodes. 
TABLE I.

Number of Primary Operations on Benign Parotid Tumors in the Period 2000 to 2008

\begin{tabular}{lccc}
\hline Year & Total No. of Operations & No. of ED & $\%$ ED \\
\hline 2000 & 69 & 9 & 13.0 \\
2001 & 69 & 18 & 26.1 \\
2002 & 110 & 27 & 24.5 \\
2003 & 84 & 28 & 33.3 \\
2004 & 85 & 38 & 44.7 \\
2005 & 104 & 54 & 51.9 \\
2006 & 123 & 61 & 49.6 \\
2007 & 152 & 64 & 42.1 \\
2008 & 138 & 78 & 56.5 \\
Total & 934 & 377 & 40.4 \\
\hline \hline & ED = extracapsular dissection.
\end{tabular}

\section{Postoperative Complications}

In the postoperative phase following extracapsular dissection 18 patients $(4.8 \%)$ developed a fluid accumulation in the form of a seroma. In most cases this was successfully drained by spreading of the wound edges (Table III). In 13 patients $(3.5 \%)$ a hematoma developed in the area of the wound. The presence of a seroma or hematoma was diagnosed on the basis of sonography. In addition to drainage, treatment consisted of daily antiseptic irrigation, application of a pressure bandage, and occasionally (where there was evidence of an inflammatory process) systemic antibiotic therapy. A salivary fistula, that is, prolonged flow of clear saliva from the wound area, developed in eight patients $(2.1 \%)$. In these cases systematic use of a pressure bandage was generally supplemented by transdermal administration of scopolamine to reduce the secretion of saliva. Secondary bleeding from the area of the operation occurred in three patients $(0.8 \%)$.

The most commonly reported unwanted effect of the operation was hypoesthesia of the cheek or earlobe. This was reported by 38 patients $(10.1 \%)$ and was most pronounced in the immediate postoperative period, after which it generally subsided markedly over a period of weeks. Lasting disturbances of sensation were reported in only four patients (1\%) and were perceived by the

TABLE II.

Histological Classification of Benign Lesions of the Parotid Gland Removed by Extracapsular Dissection.

\begin{tabular}{lrr}
\hline Histological Entity & No. & $\%$ \\
\hline Pleomorphic adenoma & 124 & 32.9 \\
Whartin's tumor & 128 & 34.0 \\
Basal cell adenoma & 8 & 2.1 \\
Other benign tumors & 36 & 9.5 \\
Cyst & 36 & 9.5 \\
Lymph node & 27 & 7.2 \\
Sialadenitis & 12 & 3.2 \\
Other histology & 6 & 1.6 \\
Total & 377 & 100 \\
\hline \hline
\end{tabular}

TABLE III.

Early Complications of Extracapsular Dissection (Excluding Facial Nerve Paresis).

\begin{tabular}{lrr}
\hline Complication & No. & $\%$ \\
\hline Seroma & 18 & 4.8 \\
Hematoma & 13 & 3.5 \\
Fistula & 8 & 2.1 \\
Secondary bleeding & 3 & 0.8 \\
Disturbance of sensation & 38 & 10.1 \\
Total & 80 & 21.2 \\
\hline
\end{tabular}

patients concerned to be scarcely troublesome or not troublesome at all.

\section{Facial Nerve Function}

Preoperatively, all patients showed normal facial nerve function corresponding to grade I of the HouseBrackmann scale. Postoperatively, facial nerve function remained unimpaired in 346 patients (91.8\%); however, 31 patients $(8.2 \%)$ showed facial nerve weakness immediately after the operation (Table IV). Most cases of paresis $(87.1 \%)$ were of House-Brackmann grade II and a few $(12.9 \%)$ of House-Brackmann grade III. No highergrade functional impairment was observed.

In 23 of the affected patients the facial nerve paresis resolved completely over a period of between 14 days and 3 months, that is, unimpaired facial nerve function (House-Brackmann I) was restored. The proportion of patients who developed temporary facial nerve paresis was thus $6.1 \%$ (Table V). The other eight patients showed persistent impairment of facial nerve function, that is, $2.1 \%$ of all the patients developed permanent facial nerve paresis. Seven of these patients had weakness only of the marginal branch of the facial nerve, corresponding to House-Brackmann grade II paresis, whereas one patient had House-Brackmann grade III paresis (Table VI). It must be pointed out, however, that in three of these patients the time between the operation and data analysis was less than 12 months (6-9 months); therefore, a final assessment was not possible in these cases. Only the remaining five patients $(1.3 \%)$ still showed facial nerve dysfunction several years after the operation.

\section{DISCUSSION}

Benign tumors of the parotid gland are a common condition with which patients present to ear, nose, and throat departments. Treatment generally consists of

TABLE IV.

Facial Nerve Function Immediately After Extracapsular Dissection.

\begin{tabular}{lrr}
\hline Facial Nerve Function Postoperatively & No. & $\%$ \\
\hline House I & 346 & 91.8 \\
House II & 28 & 7.4 \\
House III & 3 & 0.8 \\
House IV-VI & 0 & 0 \\
Total & 377 & 100 \\
\hline
\end{tabular}

Klintworth et al.: Complications After ED of Parotid Tumors 
TABLE V.

Temporary Facial Nerve Paresis After Extracapsular Dissection.

\begin{tabular}{lrc}
\hline Temporary Facial Nerve Paresis & No. & $\%$ \\
\hline House II & 21 & 5.57 \\
House III & 2 & 0.53 \\
Total & 23 & 6.10 \\
\hline \hline
\end{tabular}

surgical removal of the tumor. An important consideration in this regard is that the operation should ensure complete removal of the tumor so as to minimize the risk of recurrence and spare the patient from additional-and potentially more extensive-operations. At the same time, the surgical technique chosen should be one that achieves the best possible postoperative outcome with little or no negative impact on the patient's quality of life (given that the disease being treated is benign). In relation to parotid surgery this means above all that the function of the facial nerve must not be impaired and that the wound must heal without complications.

The rate of temporary postoperative paresis of the facial nerve is reported as $15 \%$ to $25 \%$ after superficial parotidectomy and $20 \%$ to $50 \%$ after total parotidectomy, whereas the rate of permanent facial nerve paresis is reported as $5 \%$ to $10 \%$. ${ }^{9,11,16,17,19}$

In 2006 Guntinas-Lichius et al. published a large study on the results obtained after operations on benign parotid tumors. ${ }^{19}$ They reported on 963 operations, of which $61 \%$ were superficial and $39 \%$ total parotidectomies. Temporary facial nerve paresis was found postoperatively in $22 \%$ and $30 \%$ of patients after superficial and total parotidectomy, respectively. In $17 \%$ of these patients the degree of paresis was House-Brackmann grade II, in 5\% House-Brackmann grade III/IV, and in $4 \%$ House-Brackmann grade V/VI (i.e., complete paralysis). Permanent facial nerve paresis developed in $6 \%$ of all patients who underwent surgery without distinction between the various surgical techniques. Permanent House-Brackmann grade II paresis developed in 3.6\%, House-Brackmann grade III in $1.7 \%$, House-Brackmann grade IV in $0.6 \%$, and House-Brackmann grade $\mathrm{V}$ in $0.2 \%$ of all patients.

In 2007 Zernial et al. reported on 73 cases of pleomorphic adenoma, of which 42 were successfully followed up. ${ }^{11}$ Temporary facial nerve paresis occurred in $17.9 \%$ of patients after superficial parotidectomy and in as many as $43.8 \%$ of patients after total parotidectomy. Amazingly, facial nerve function recovered completely in all the patients who were successfully followed up.

The literature contains many reports with similar results to these. For example, in 2007 Roh et al. reported a rate of $34.7 \%$ for the occurrence of temporary facial nerve paresis after superficial or total parotidectomy in 49 patients. ${ }^{10}$ Temporary facial nerve paresis is, therefore, to be expected in about one fifth of patients after superficial parotidectomy and in one third to one half of patients after total parotidectomy.
On the other hand, there is evidence in the literature to suggest that the risk of temporary or permanent facial nerve paresis is significantly less after extracapsular dissection than after superficial or total parotidectomy. ${ }^{9,13,16}$

In 1996 McGurk et al. reported on 475 patients who had undergone surgery for pleomorphic adenoma. ${ }^{16}$ After extracapsular dissection $(n=380) 11 \%$ of patients showed temporary and $2 \%$ permanent facial nerve paresis, whereas after superficial parotidectomy $(\mathrm{n}=95)$ the corresponding figures were $32 \%$ and $1 \%$, respectively, although it must be noted that no facial nerve monitoring was performed intraoperatively in these patients. In this study the risk of temporary facial nerve dysfunction was almost three times higher after the more invasive procedure than after circumscribed resection. A similar result was obtained in the study by Roh et al. ${ }^{10}$ referred to above; temporary facial nerve paresis occurred in $34.7 \%$ of patients after superficial parotidectomy (see above) but only in $11.5 \%$ of patients after partial parotidectomy, whereas permanent paresis occurred only after superficial parotidectomy $(2 \%)$.

Rehberg et al. reported on the results of 348 primary operations for benign parotid tumors. ${ }^{9}$ The rate of temporary facial nerve paresis was $1.9 \%$ after partial parotidectomy, $22.0 \%$ after superficial parotidectomy, and $46.7 \%$ after total parotidectomy, whereas the rate of persistent facial nerve dysfunction was $0.7 \%$ after partial, $2.0 \%$ after superficial, and $10.0 \%$ after total parotidectomy. Here again, therefore, it was found that greater invasiveness of the procedure performed was associated with a markedly higher risk of damage to the facial nerve.

Like facial nerve paresis, Frey's syndrome also occurs far less commonly after extracapsular dissection than after the routinely performed procedures of superficial or total parotidectomy. Thus, in the study referred to above, McGurk et al. found an incidence of Frey's syndrome of $38 \%$ after superficial parotidectomy compared to only $5 \%$ after extracapsular dissection. ${ }^{16}$ Hancock reported an incidence of Frey's syndrome of $25 \%$ after superficial parotidectomy, ${ }^{12}$ whereas Prichard et al. found an incidence as high as $40 \%,{ }^{13}$ and neither of these authors observed any cases of Frey's syndrome after extracapsular dissection. The lower incidence of gustatory sweating after extracapsular dissection is presumably due mostly to the fact that with this technique less glandular tissue has to be resected. ${ }^{15,20}$ However, because this phenomenon is a late rather than an early complication, it will not be discussed in any more detail here.

The main argument raised against techniques of circumscribed partial resection, such as extracapsular

TABLE VI.

Permanent Facial Nerve Paresis After Extracapsular Dissection.

\begin{tabular}{lcc}
\hline Permanent Facial Nerve Paresis & No. & $\%$ \\
\hline House II & 7 & 1.86 \\
House III & 1 & 0.27 \\
Total & 8 & 2.12 \\
\hline \hline
\end{tabular}

Klintworth et al.: Complications After ED of Parotid Tumors 
dissection and partial parotidectomy, is the supposedly higher recurrence rate that these techniques are commonly postulated to have. ${ }^{5,7}$ In this context a clear distinction must be made between the modern technique of extracapsular dissection and the historical technique of enucleation, with which recurrence rates of $20 \%$ to $40 \%$ have been reported. ${ }^{16,20}$

Especially in the case of pleomorphic adenomas, it is also argued that a ruptured or incomplete capsule and the presence of pseudopodia or satellite nodules can be responsible for recurrences if the tumor capsule is exposed during an operation or if the tumor is removed with only a narrow margin of normal glandular tissue rather than, for example, being removed en bloc with the entire superficial lobe of the gland. ${ }^{19,21}$ On the other hand, a number of studies have challenged this theory. For example, Donovan und Conley found that in $60 \%$ of supposedly en bloc resections performed as part of superficial parotidectomy the tumor capsule was partially exposed and dissected because the tumor abutted the facial nerve. ${ }^{22}$ Moreover, in $21 \%$ of cases the tumor extended to the edge of the histological specimen, and in a further $40 \%$ of cases only an extremely narrow resection margin was present. Despite this, no increase in recurrence rate could be demonstrated in the patients concerned.

Ghosh et al. analyzed a series of 83 pleomorphic adenomas re-evaluating the histological slides to define risk factors for recurrence. ${ }^{23}$ After a mean follow-up of 12.5 years, they found a recurrence rate of $17.6 \%$ in cases where tumor cells were present at the margin, but only of $1.8 \%$ if they were found within $1 \mathrm{~mm}$ of the margin but not directly at it. Therefore, they conclude that a layer of connective tissue consisting of merely one or two cell rows is sufficient to prevent recurrence.

In a retrospective analysis of histological specimens published in 2002, Witt found that both extracapsular dissection and superficial and total parotidectomy almost always resulted in focal capsular exposure, and that the recurrence rate did not differ between the various techniques. $^{17}$

A number of studies have already shown that the recurrence rate of pleomorphic adenomas is no higher with extracapsular dissection than with superficial or total parotidectomy. $9,12,16,17$ For example, after a mean follow-up period of 12.5 years, McGurk et al. found a recurrence rate of $2 \%$ both with extracapsular dissection $(\mathrm{n}=380)$ and with superficial parotidectomy $(\mathrm{n}=95){ }^{16}$ Rehberg et al. reported recurrence rates of $2.3 \%$ after extracapsular dissection, $0 \%$ after superficial parotidectomy, and $15.4 \%$ after total parotidectomy. ${ }^{9}$ Neither Hancock $^{12}$ nor Smith et al. ${ }^{20}$ observed any recurrences after extracapsular dissection in 42 and 27 patients, respectively. The recurrence rate reported with extracapsular dissection is thus similar to that reported with superficial and total parotidectomy, namely $0 \%$ to $5 \% .^{8,9,16,19}$ A preliminary analysis of our own (as yet unpublished) data also suggests that extracapsular dissection does not increase the recurrence rate as we have not encountered any recurrence of a tumor that had been removed by this technique (as a primary intervention) so far.

\section{CONCLUSION}

In benign tumors of the parotid gland extracapsular dissection represents a surgical alternative to the in many cases routinely performed techniques of superficial or total parotidectomy in case of a lesion that appears mobile and is located in the superficial lobe of the gland. Facial nerve monitoring must be used obligatorily.

An analysis of our data confirms the findings in the literature that the risk for postoperative complications is markedly less with this technique than with more invasive interventions. In particular, postoperative facial nerve dysfunction is far less likely to occur. At the same time, it is yet to be proven that techniques of circumscribed partial resection of the parotid gland, such as extracapsular dissection, increase the recurrence rate of pleomorphic adenoma; on the contrary, the published data clearly refute this hypothesis.

It should also be borne in mind that in the event that a revision operation becomes necessary, better conditions, especially for dissection of the facial nerve, are present after extracapsular dissection than after superficial or total parotidectomy, because with extracapsular dissection the nerve is not exposed in the course of the primary operation and consequently no scar tissue is present, which can make dissection much more difficult at the revision operation.

We therefore recommend that superficial parotidectomy no longer be regarded as the minimal intervention required for the removal of benign parotid tumors, but rather that extracapsular dissection be included in the range of surgical techniques available for this purpose.

\section{BIBLIOGRAPHY}

1. Sungur N, Akan IM, Ulusoy MG, Ozdemir R, Kilinc H, Ortak T. Clinicopathological evaluation of parotid gland tumors: a retrospective study. J Craniofac Surg 2002;13: 26-30.

2. Benedict EB. Tumors of the parotid gland: a study of two hundred and twenty-five cases with complete end-results in eighty cases. Surg Gynecol Obstet 1930;51:626-647.

3. Rawson AJ, Howard JM, Royster HP, Horn RC Jr. Tumors of the salivary glands; a clinicopathological study of 160 cases. Cancer 1950;3:445-458.

4. McFarland J. Three hundred mixed tumors of the salivary glands of which 69 recurred. Surg Gynecol Obstet 1936; 63:457-468.

5. Patey DH, Thackray AC. The treatment of parotid tumours in the light of a pathological study of parotidectomy material. Br J Surg 1958;45:477-487.

6. Janes RM. The treatment of tumors of the salivary gland by radical excision. Can Med Assoc $J$ 1940;43:554-559.

7. Guntinas-Lichius O, Kick C, Klussmann JP, Jungehuelsing M, Stennert E. Pleomorphic adenoma of the parotid gland: a 13-year experience of consequent management by lateral or total parotidectomy. Eur Arch Otorhinolaryngol 2004;261:143-146.

8. Laccourreye H, Laccourreye O, Cauchois R, Jouffre V, Menard M, Brasnu D. Total conservative parotidectomy for primary benign pleomorphic adenoma of the parotid gland: a 25-year experience with 229 patients. Laryngoscope 1994;104:1487-1494.

9. Rehberg E, Schroeder HG, Kleinsasser O. Surgery in benign parotid tumors: individually adapted or standardized radical interventions [in German]? Laryngorhinooto logie 1998;77:283-288. 
10. Roh JL, Kim HS, Park CI. Randomized clinical trial comparing partial parotidectomy versus superficial or total parotidectomy. Br J Surg 2007;94:1081-1087.

11. Zernial O, Springer IN, Warnke P, Harle F, Risick C, Wiltfang J. Long-term recurrence rate of pleomorphic adenoma and postoperative facial nerve paresis (in parotid surgery). J Craniomaxillofac Surg 2007;35:189-192.

12. Hancock BD. Clinically benign parotid tumours: local dissection as an alternative to superficial parotidectomy in selected cases. Ann R Coll Surg Engl 1999;81:299-301.

13. Prichard AJ, Barton RP, Narula AA. Complications of superficial parotidectomy versus extracapsular lumpectomy in the treatment of benign parotid lesions. $J R$ Coll Surg Edinb 1992;37:155-158.

14. Gleave EN, Whittaker JS, Nicholson A. Salivary tumoursexperience over thirty years. Clin Otolaryngol Allied Sci 1979;4:247-257.

15. McGurk M, Thomas BL, Renehan AG. Extracapsular dissection for clinically benign parotid lumps: reduced morbidity without oncological compromise. $\mathrm{Br}$ J Cancer 2003; 89:1610-1613.

16. McGurk M, Renehan A, Gleave EN, Hancock BD. Clinical significance of the tumour capsule in the treatment of parotid pleomorphic adenomas. Br J Surg 1996;83:1747-1749.
17. Witt RL. The significance of the margin in parotid surgery for pleomorphic adenoma. Laryngoscope 2002;112: $2141-2154$.

18. House JW, Brackmann DE. Facial nerve grading system. Otolaryngol Head Neck Surg 1985;93:146-147.

19. Guntinas-Lichius O, Klussmann JP, Wittekindt C, Stennert E. Parotidectomy for benign parotid disease at a university teaching hospital: outcome of 963 operations. Laryngoscope 2006;116:534-540.

20. Smith SL, Komisar A. Limited parotidectomy: the role of extracapsular dissection in parotid gland neoplasms. Laryngoscope 2007;117:1163-1167.

21. Zbaren P, Stauffer E. Pleomorphic adenoma of the parotid gland: histopathologic analysis of the capsular characteristics of 218 tumors. Head Neck 2007;29: 751-757.

22. Donovan DT, Conley JJ. Capsular significance in parotid tumor surgery: reality and myths of lateral lobectomy. Laryngoscope 1984;94:324-329.

23. Ghosh S, Panarese A, Bull PD, Lee JA. Marginally excised parotid pleomorphic salivary adenomas: risk factors for recurrence and management. A 12.5-year mean follow-up study of histologically marginal excisions. Clin Otolaryngol Allied Sci 2003;28:262-266. 